\title{
Femtosecond Yb:YCOB laser pumped by narrow-stripe laser diode and passively modelocked using ion implanted saturable-absorber mirror
}

Citation for published version (APA):

Valentine, G. J., Kemp, A. J., Birkin, D. J. L., Burns, D., Balembois, F., Georges, P., Bernas, H., Aron, A., Aka, G., Sibbett, W., Brun, A., Dawson, M. D., \& Bente, E. A. J. M. (2000). Femtosecond Yb:YCOB laser pumped by narrow-stripe laser diode and passively modelocked using ion implanted saturable-absorber mirror. Electronics Letters, 36(19), 1621-1623. https://doi.org/10.1049/el:20001141

DOI:

10.1049/el:20001141

Document status and date:

Published: 01/01/2000

\section{Document Version:}

Publisher's PDF, also known as Version of Record (includes final page, issue and volume numbers)

\section{Please check the document version of this publication:}

- A submitted manuscript is the version of the article upon submission and before peer-review. There can be important differences between the submitted version and the official published version of record. People interested in the research are advised to contact the author for the final version of the publication, or visit the DOI to the publisher's website.

- The final author version and the galley proof are versions of the publication after peer review.

- The final published version features the final layout of the paper including the volume, issue and page numbers.

Link to publication

\footnotetext{
General rights

- You may freely distribute the URL identifying the publication in the public portal. follow below link for the End User Agreement:

www.tue.nl/taverne

Take down policy

If you believe that this document breaches copyright please contact us at:

openaccess@tue.nl

providing details and we will investigate your claim.
}

Copyright and moral rights for the publications made accessible in the public portal are retained by the authors and/or other copyright owners and it is a condition of accessing publications that users recognise and abide by the legal requirements associated with these rights.

- Users may download and print one copy of any publication from the public portal for the purpose of private study or research.

- You may not further distribute the material or use it for any profit-making activity or commercial gain

If the publication is distributed under the terms of Article $25 \mathrm{fa}$ of the Dutch Copyright Act, indicated by the "Taverne" license above, please 
in the quantum efficiency of the laser. Indeed, after the forward bias ESD stress test an inhomogeneous surface layer morphology was observed in some cases, in contrast to the unstressed samples.
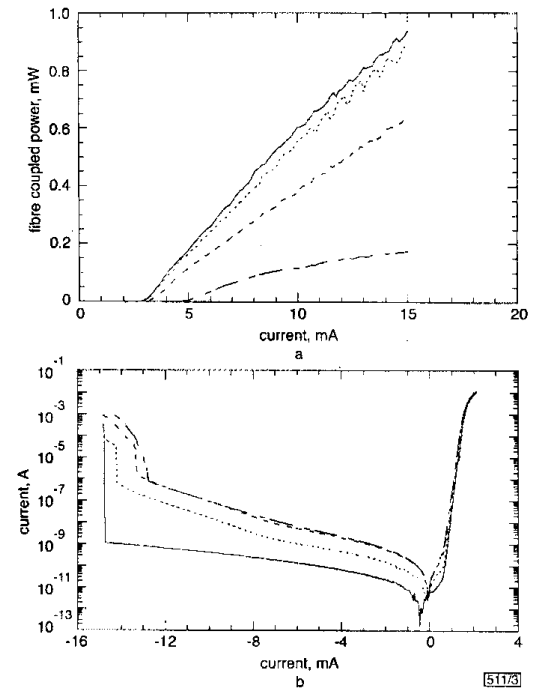

Fig. 3 Results of reverse bias ESD step stress test of proton implanted $V C S E L$

$a$ Optical power against current

$b$ Current-voltage characteristics before and at different steps

before ESD - . . - $-1200 \mathrm{~V}$

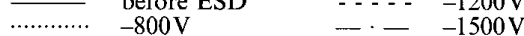

At increased forward bias stress amplitudes, the heating of the surface region induces propagation of near-surface defects, e.g. originating from the proton implantation [5], towards the active region. Measurements of the optical emission spectra during ESD tests did not show significant changes in the emission wavelength at the beginning of the degradation process. This indicates that the active layer is not affected in this stage of degradation. However, when the ESD stress test was continued to pulse amplitudes well above the damage threshold value, lasing was completely suppressed and a broad, LED-type, emission spectrum was observed.

The development of the $P-I$ characteristics during negative bias ESD stress (Fig. $3 a$ ) is very similiar to the development observed during forward bias stress (Fig. 2a), also the quantum efficiency drops initially and only later does the lasing threshold current increase. In the development of the $I-V$ characteristics, additional to the sudden increase of the dark current after degradation there is also a monotonically decreasing breakdown voltage from an initial value of $-14.8 \mathrm{~V}$ before degradation to a value of $-13.0 \mathrm{~V}$ after application of the $-1500 \mathrm{~V}$ ESD pulse.

It can be concluded that, in particular, the positive bias stress ESD damage threshold values of proton implanted VCSELs are substantially lower than the values reported for 820 and $1320 \mathrm{~nm}$ LEDs [6] and $1300 \mathrm{~nm}$ Fabry-Perot laser diodes [7], devices frequently used in optical access network and LAN applications.

(C) IEE 2000

Electronics Letters Online No: 20001152

1 August 2000

DOI: 10.1049/el:20001152

H.C. Neitzert (Università di Salerno, Dipartimento di Elettronica, I84084 Fisciano (SA), Italy)

\section{References}

1 FIEDLER, U., REINER, G., SCHNITZER, P., and EBELING, K.J.: 'Top surface-emitting vertical-cavity laser diodes for $10-\mathrm{Gb} / \mathrm{s}$ data transmission', IEEE Photonics Technol. Lett., 1996, 8, pp. 746-748

2 HAWTHORNE, R.A., GUENTER, J.K., GRANVILLE, D.N., HIBBS-BRENNER, M.K., and MORGAN, R.A.: 'Reliability study of $850 \mathrm{~nm}$ VCSELs for data communications'. Proc. 34th IEEE Rel. Symp., 1996, pp. 203 210

3 CHENG, Y.M., HERRICK, R., PETROFF, P.M., HIBBS-BRENNER, M.K., and MORGAN, R.A.: 'Degradation mechanisms of vertical cavity surface emitting lasers'. Proc. 34th IEEE Rel. Symp., 1996, pp. 211-213

4 ESD STM5.1: 'Sensitivity testing, Human Body Model, component level'. ESD Association, Rome, 1998
5 JIANG, W., GAW, C., KIELY, P., LAWRENCE, B., LEBBY, M., and CLAISSE, P.R.: 'Effect of proton implantation on the degradation of GaAs/GaAlAs vertical cavity surface emitting lasers', Electron. Lett., 1997, 33, pp. 137-139

6 NEITZERT, H.C., and PICCIRILLO, A.: 'Sensitivity of multimode bidirectional optoelectronic modules to electrostatic discharges', Microelectron. Reliab., 1999, 39, pp. 1863-1871

7 TWU, Y., CHENG, L.S., CHU, S.N.G., NASH, F.R., WANG, K.W., and PARAYANTHAL, P.: 'Semiconductor laser damage due to humanbody-model electrostatic discharge', J. Appl. Phys., 1993, 74, pp. $1510-1520$

\section{Femtosecond Yb:YCOB laser pumped by narrow-stripe laser diode and passively modelocked using ion implanted saturable-absorber mirror}

G.J. Valentine, A.J. Kemp, D.J.L. Birkin, D. Burns, F. Balembois, P. Georges, H. Bernas, A. Aron, G. Aka, W. Sibbett, A. Brun, M.D. Dawson and E. Bente

The authors present, what they believe to be, the first femtosecond Yb:YCOB laser, pumped by a low-power, narrowstripe laser diode. To facilitate modelocking, high-energy oxygen ion implantation of the saturable absorber is employed. $210 \mathrm{fs}$ pulse generation at $16 \mathrm{~mW}$ average output power for $140 \mathrm{~mW}$ incident pump power is reported.

Recently, we reported on the possibility of using low-power, narrow-stripe AlGaInP laser diodes as the pump source for femtosecond Cr:LiSAF lasers [1]. This is a low-cost alternative to highpower broad-stripe laser-diode pump sources since the power available from narrow-stripe devices, though lower, is used more efficiently owing to their near-diffraction-limited beams. This technique may be appropriate to laser systems for which higher power narrow-stripe laser diodes are available, e.g. $1 \mu \mathrm{m}$ ytterbium based lasers pumped at the zero-phonon line near $976 \mathrm{~nm}$. In this spectral region, narrow-stripe InGaAs laser diodes are available with powers as high as $200 \mathrm{~mW}$.

One promising ytterbium host which has recently received interest is the calcium rare-earth oxoborate matrix (i.e. $\mathrm{Ca}_{4} \mathrm{YO}\left(\mathrm{BO}_{3}\right)_{3}$ (YCOB) or $\mathrm{Ca}_{4} \mathrm{GdO}\left(\mathrm{BO}_{3}\right)_{3}(\mathrm{GdCOB})$ ) [2]. Its strong crystal field minimises the quasi-three-level behaviour of $\mathrm{Yb}$ (due to the large splitting of the ground state) and broadens the emission band permitting ultrashort pulse generation. As a potential medium for the development of an efficient, low-cost, low-power laser system, $\mathrm{Yb}: \mathrm{YCOB}$ appears to be very promising: when pumped at the zero-phonon line at $976 \mathrm{~nm}$, the quantum defect is less than $7 \%$ [2] and its gain-cross-section upperstate-lifetime product is four times larger than Cr:LiSAF and ten times that of Yb:glass.

Unfortunately, the small gain-cross-section and long upperstatelifetime of $\mathrm{Yb}: \mathrm{YCOB}$ contribute to a high threshold for stable continuous wave $(\mathrm{CW})$ modelocking [3]. Although a $90 \mathrm{fs} \mathrm{Yb}$ :GdCOB laser was recently reported [4], it used a semiconductor saturable absorber mirror as the modelocking device which incorporated an absorber grown at low-temperature to increase defects and hence reduce the absorber recovery time [3]. Because this device was prone to damage by a Q-switching Yb laser, our present work has concentrated on using saturable Bragg reflectors (SBR) grown at optimum temperature. These have high damage thresholds due to few defects but hence have a long absorber recovery time. This further increases the threshold for CW modelocking.

In this Letter we report on a first experiment to assess the feasibility of modelocking an $\mathrm{Yb}: \mathrm{YCOB}$ laser system pumped by one narrow-stripe laser diode. Ion implantation of a metal-organic chemical vapour deposition (MOCVD) grown SBR is utilised to increase the defect concentration and hence reduce the absorber recovery time to facilitate modelocking. This post-growth defect enhancement approach offers the possibility of 'tailoring' SBR absorbers to specific applications.

Fig. 1 shows a schematic diagram of the cavity used, which is similar to the laser from [1]. The folding mirror through which the pump was focused (M1) was operated close to normal incidence. The astigmatism from the Brewster cut gain medium was compen- 
sated by the larger angle of the short arm folding mirror (M2). This allows tight focusing on the pumped end of the Brewster-cut rod. For optimised CW pumping and subsequent adaptation to SBR assisted modelocking, the long arm of the cavity was set to $\sim 1.5 \mathrm{~m}$ and the short arm to $10 \mathrm{~cm}$. This created a beam waist on the end of the short arm of $\sim 17 \mu \mathrm{m}$ suitable for tight focusing onto a SBR, and a waist of $29 \mu \mathrm{m} \times 17 \mu \mathrm{m}$ in the gain medium.

The available gain medium was a $4 \mathrm{~mm}$ long Brewster cut crystal with $15 \% \mathrm{Yb}$, having a maximum pump absorption coefficient of $6 \mathrm{~cm}^{-1}$ at $976 \mathrm{~nm}$. No active cooling was employed.

The pump source was a single, narrow-stripe InGaAs laser diode supplied by Uniphase, rated at a maximum output power of $180 \mathrm{~mW}$ at $976 \mathrm{~nm}$ with a spectral width of $<1 \mathrm{~mm}$. The emitting aperture was $\sim 3 \mu \mathrm{m} \times 1 \mu \mathrm{m}$, giving a near-diffraction limited, though asymmetric, beam. $6.2 \mathrm{~mm}$ aspheric and $7.5 \mathrm{~cm}$ plano-convex lenses served to collect and focus the beam into the gain medium. No reshaping of the asymmetric beam was performed.

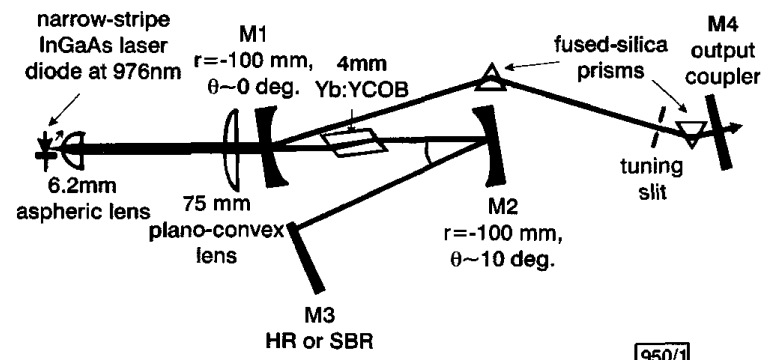

Fig. 1 Schematic diagram of low-threshold modelocked $Y b: Y C O B$ laser (not to scale)

The use of diffraction limited laser diodes offers two advantages compared to higher power broad-stripe devices. First, the superior beam quality allows increased gain saturation by permitting much tighter laser and pump focusing while maintaining excellent laserpump mode overlap. This reduces the threshold for $\mathrm{CW}$ modelocking. Secondly, the narrower emission spectrum has an enhanced overlap with the narrow ( $2.3 \mathrm{~nm}$ FWHM) zero-line absorption band at $976 \mathrm{~nm}$ for a higher effective pump absorption coefficient.

For passive modelocking, a MOCVD grown SBR was utilised comprising a $10 \mathrm{~nm}$-thick InGaAs quantum well, situated in the middle of the top $\mathrm{GaAs}$ layer of a GaAs/AlGaAs Bragg mirror stack. As grown, this structure contained few defects and hence had a long absorber recovery time [3]. To shorten this subsequent to growth, a strip of this SBR was subjected to high-energy ion bombardment [5]. Nickel (Ni) and oxygen (O) ion bombardment was performed at energies of $12 \mathrm{MeV}$ and $9.5 \mathrm{MeV}$ respectively, both to a density of $10^{12} \mathrm{~cm}^{-2}$. This was expected to increase the defect density of the absorber and hence reduce the absorber recovery time. Unlike low-energy ion implantation techniques which have been previously reported to successfully reduce the recovery time of saturable absorbers by implanting defect species directly into the absorber layer [6], high-energy ions pass straight through this layer, finally stopping within the Bragg mirror/substrate, leaving behind a trail of defects. This technique has been successfully demonstrated in InGaAs multiple quantum well absorbers for pulse cleaning in telecommunications applications [5]. We believe this to be the first application of high-energy ion implantation to a saturable absorber on a mirror structure.

Initially, the laser was aligned with all HR coated mirrors and optimised for a low CW threshold. Laser oscillation was achieved for incident pump powers below $12 \mathrm{~mW}$. After re-alignment at the maximum incident pump power of $150 \mathrm{~mW}$, the optimum output coupling of $1 \%$ gave an output power of $45 \mathrm{~mW}$, a slope efficiency of $37 \%$ and a corresponding threshold of $35 \mathrm{~mW}$. These are encouraging first results considering that the long free-running wavelength of $1050 \mathrm{~nm}$ indicates re-absorption loss at shorter wavelengths and is evidence that this gain medium is too long.

For modelocked operation, M3 was replaced by the implanted SBR. The CW laser output power with a $1 \%$ output coupler fell from $45 \mathrm{~mW}$ (with HR mirror) to $39 \mathrm{~mW}$ for the O implanted sample but down to $11 \mathrm{~mW}$ for $\mathrm{Ni}$ indicating that high-energy $\mathrm{Ni}$ implantation had significantly increased the nonsaturable losses of the SBR. No modelocking was subsequently observed with the $\mathrm{Ni}$ implanted sample.
Dispersion compensation, provided by two low loss fused-silica prisms separated by $67 \mathrm{~cm}$, permitted femtosecond soliton modelocked operation. Using the O implanted SBR, CW modelocked operation was readily obtained at maximum pump power by tuning the laser through the absorption band edge near $1050 \mathrm{~nm}$; however, it was characterised by a broad, multiple lobed, unstable spectrum indicating multiple pulsed modelocked behaviour. Further optimisation of the prism glass insertion, pump power reduction and realignment of the cavity permitted stable, single pulsed modelocked operation at a repetition rate of $\sim 85 \mathrm{MHz}$. A pulse duration of $210 \mathrm{fs}$ was deduced from the intensity autocorrelation in Fig. $2 a$ assuming a sech ${ }^{2}$ profile. The corresponding spectrum in Fig. $2 b$ verifies the pulses to be near-transform limited $(\Delta v \Delta \tau=$ 0.3 ) with a central wavelength of $1052 \mathrm{~nm}$. The modelocked output power was $16 \mathrm{~mW}$ through a $1 \%$ output coupler for $140 \mathrm{~mW}$ incident pump power. Increasing the pump power and realigning the cavity for maximum output power resulted in unstable multiple pulsed operation. With further optimisation of this cavity, higher modelocked output powers and shorter pulse durations should be possible

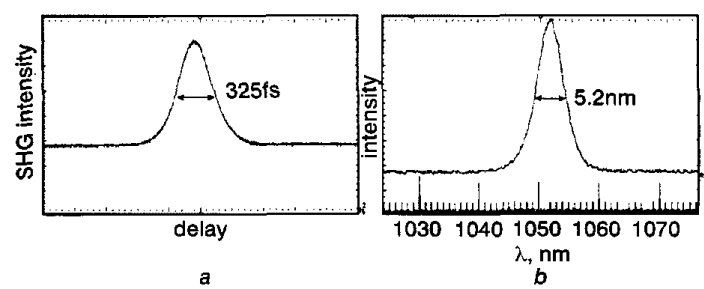

Fig. 2 Temporal and spectral characteristics of $210 \mathrm{fs}$ pulses

a co-linear intensity autocorrelation

$b$ corresponding spectrum

Using an unimplanted SBR allowed the importance of the implantation to be qualitatively assessed. Unlike the implanted sample, strong Q-switched behaviour predominated as the laser was tuned through the absorption band. With great difficulty, only unstable, multiple pulse modelocking was briefly observed; no stable single pulse modelocking could be achieved for any output power, indicating clearly that implantation was crucial for the modelocking of this $\mathrm{Yb}: \mathrm{YCOB}$ laser system. Of course, further investigations into the effects of the high-energy implantation on the absorber dynamics as well as optimisation of the implantation level for modelocking will need to be carried out.

In summary, we have demonstrated, for the first time to our knowledge, a femtosecond $\mathrm{Yb}$ :YCOB laser. A narrow-stripe laser diode provided the pump power and modelocking was established using an ion-implanted SBR. High-energy ion implantation has been utilised for the reduction of the absorber recovery time to facilitate modelocking of gain media with very high gain saturation intensities. Optimisation of the intracavity dispersion is expected to further reduce the pulse durations generated by this modelocked laser. Although a reduction of the gain medium length will improve the $\mathrm{CW}$ performance reported, this work has shown the approach of using lower power, but superior beam quality, narrow-stripe InGaAs laser diode pump sources to be very promising for femtosecond $\mathrm{Yb}$ based lasers.

Acknowledgment: The CNRS (Action Conciertée Optique programme), CNRS/Royal Society Cooperation and TMR 'NOMOKE' are acknowledged for financial support.

(C) IEE 2000

30 June 2000

Electronics Letters Online No: 20001141

DOI: 10.1049/el:20001141

G.J. Valentine, F. Balembois, P. Georges, and A. Brun (Laboratoire Charles Fabry de l'Institut d'Optique, Université Paris Sud, Bat 503, BP 147, 91403 Orsay, France)

E-mail: gv0@hotmail.com

$\mathrm{H}$. Bernas (Centre de Spectrométrie Nucléaire et de Spectrométrie de Masse, Université Paris Sud, Bat 104, 91405 Orsay, France)

A. Aron and G. Aka (Ecole Nationale Supérieure de Chimie de Paris, Paris, France) 
A.J. Kemp, D.J.L. Birkin and W. Sibbett (School of Physics and Astronomy, University of St Andrews, North Haugh, St Andrews, Fife KY16 9SS, United Kingdom)

D. Burns, M.D., Dawson and E. Bente (Institute of Photonics, University of Strathclyde, Wolfson Centre, 106 Rottenrow, Glasgow G4 oNW, United Kingdom)

\section{References}

1 HOPKINS, J.-M., VALENTINE, G.J., SIBBETT, W., AUS DER AU, J., MORIERGENOUD, F., KELLER, U., and VALSTER, A.: 'Efficient, low-noise SESAM-based femtosecond $\mathrm{Cr}^{3+}: \mathrm{LiSrAlF}_{6}$ laser', Opt. Commun., 1998, 154, pp. 54-58

2 DRuon, F., AUGE, F., BALEmbois, F., GeORGES, P., Brun, A., ARON, A., MOUGEL, F., AKA, G., and VIVIEN, D.: 'Efficient, tunable, zero-linediode-pumped, continuous-wave $\mathrm{Yb}^{3+}: \mathrm{Ca}_{4} \mathrm{LnO}\left(\mathrm{BO}_{3}\right)_{3} \quad(\mathrm{Ln}=\mathrm{Gd}, \mathrm{Y})$ laser at room temperature, application to miniature lasers', J. Opt. Soc. Am. B, 2000, 17, (1), pp. 18-22

3 KELLER, U.: 'Semiconductor nonlinearities for solid-state laser modelocking and Q-switching' in KOST, A., and GARMIRE, F. (Eds.), 'Nonlinear optics in semiconductors II', Semicond. Semimet., 1999, Vol. 59, Chap. 4, pp. 211-286

4 Druon, F., Balembois, F., Georges, P., BRUN, A., COURJaud, A., HONNINGER, C., SALIN, F., ARON, A., AKA, G., and VIVIEN, D.: 'Generation of 90-fs pulses from a mode-locked diode-pumped $\mathrm{Yb}^{3+}: \mathrm{Ca}_{4} \mathrm{GdO}\left(\mathrm{BO}_{3}\right)_{3}$ laser', Opt. Lett., 2000, 25, (6), pp. 423-425

5 LUGAGNE DELPON, F., OUDAR, J.L., BOUCHE, N., RAJ, R., SHEN, A, STELMAKH, N., and LOURTIOZ, J.M.: 'Ultrafast excitonic saturable absorption in ion-implanted InGaAs/InAlAs multiple quantum wells', Appl. Phys. Lett., 1998, 72, (7), pp. 759-761

6 LEDERER, M.J., LUTHER-DAVIES, B., TAN, H.H., and JAGADISH, C.: 'An antiresonant Fabry-Perot saturable absorber for passive modelocking fabricated by metal-organic vapor phase epitaxy and ion implantation design, characterization, and mode-locking', IEEE $J$. Quantum Electron., 1998, 34, (11), pp. 2150-2161

\section{Optoelectronic feedback loop for relaxation oscillation intensity noise suppression in Tm-Ho:YAG laser}

\section{Svelto, S. Taccheo, M. Marano, G. Sorbello and P. Laporta}

An optoelectronic feedback loop that can be used to effectively reduce the intensity noise of a $2.1 \mu \mathrm{m}$ Tm-Ho:YAG laser has been designed. The feedback circuit is based on a variable-gain biquadratic bandpass filter with adjustable central frequency and quality factor, providing a high loop gain around the relaxation oscillation frequency of the laser and a closed-loop phase margin larger than $50^{\circ}$. The relaxation oscillation peak in the intensity noise spectrum was completely suppressed in closed-loop operation, and a noise reduction of up to $24 \mathrm{~dB}$ was obtained.

Introduction: CW diode-pumped Tm-Ho:YAG lasers emitting in the $2 \mu \mathrm{m}$ region are attractive sources for applications such as coherent detection of gas traces in the atmosphere and injection seeding for wind detection Doppler lidar systems [1], where low intensity noise of the laser source is a main constraint. Intensity noise in solid-state lasers is dominated by technical noise, ascribable to different sources, such as mechanical vibrations, acoustical and thermal noise and pump power fluctuations. Irrespective of the origin of the perturbation, the relaxation oscillation phenomenon [2], characterised by a strongly enhanced peak at a spectral frequency from a few tens to a few hundreds of kilohertz, is always present in the intensity noise spectrum. This noise peak, caused by the resonant behaviour of the laser response to perturbations, can be effectively reduced by means of an optoelectronic feedback loop acting on the pump power level $[3,4]$. We recently investigated the Tm-Ho system [5] and demonstrated the feasibility of intensity-noise suppression in a Tm-Ho:YAG laser [6]. In this Letter, we illustrate the design criteria of a suitable high gain feedback circuit for a $\mathrm{Tm}-\mathrm{Ho}$ laser system and report on complete suppression of the relaxation oscillation peak in the noise spectrum.
Tm-Ho:YAG laser: The laser cavity used in our experiments is a two-mirror $52 \mathrm{~mm}$ long hemispherical resonator. The active material, placed at one end of the resonator, is a $3 \mathrm{~mm}$ long YAG rod doped with $6 \% \mathrm{Tm}$ and $0.36 \%$ Ho concentrations. The laser is longitudinally pumped at $785 \mathrm{~nm}$ by a $1 \mathrm{~W}$ broad-area GaAlAs laser diode. A suitable reshaping optical system focuses the pump radiation in the active material, where an almost circular $\sim 50 \mu \mathrm{m}$ beam waist is obtained. An output power up to $60 \mathrm{~mW}$ is obtained in multi-axial-mode configuration. Two uncoated intracavity etalons, $300 \mu \mathrm{m}$ and $100 \mu \mathrm{m}$ thick, allow single-longitudinal-mode operation with an emission wavelength tunable from 2085 to $2099 \mathrm{~nm}$, and an output power up to $\sim 20 \mathrm{~mW}$.

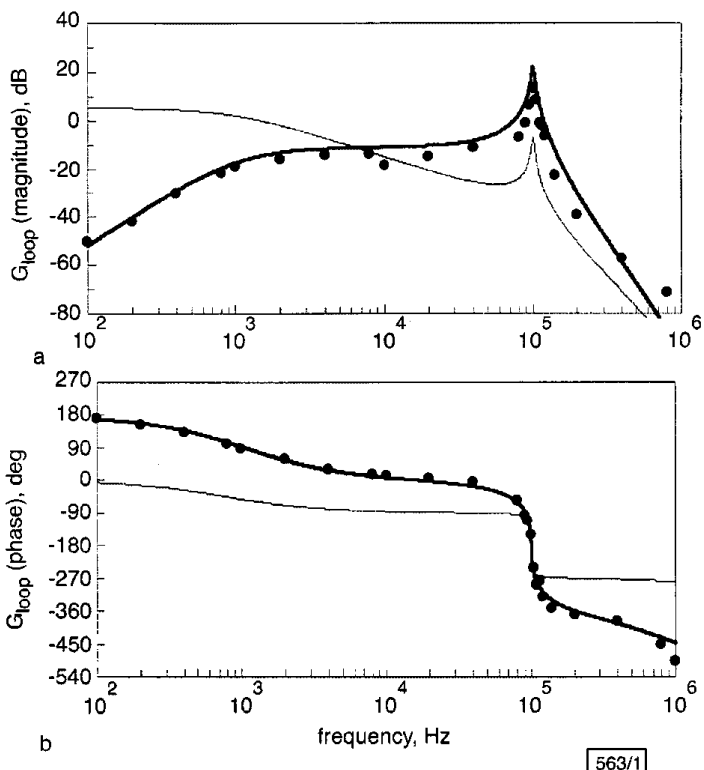

Fig. 1 Measured and calculated magnitude of loop gain magnitude and phase

$a$ Loop gain magnitude

$b$ Phase

- measured calculated calculated magnitude and phase of PTF

Pump transfer function: To design a suitable feedback circuit for intensity noise suppression, we investigated both theoretically [5] and experimentally [6] the effects of pump power fluctuations on the laser relative intensity noise (RIN). These effects can be described in the frequency domain by means of the so-called pump transfer function (PTF). We assumed a rate-equation model for the Tm-Ho system, which takes into account two intermediate steps in the pumping process (Tm-Tm cross-relaxation and TmHo energy-transfer) as well as the up-conversion processes from the upper laser level: the PTF was obtained by linearisation of the corresponding equations. Two complex-conjugate poles at the relaxation oscillation frequency ( $\mathrm{ROF}$ ), typically ranging from 50 $150 \mathrm{kHz}$, are responsible for an enhanced peak in the noise spectrum. A first step in the pumping process, namely the Tm-Tm cross-relaxation, introduces a high-frequency pole located at about a few hundreds of kilohertz, well beyond the ROF. The Tm-Ho energy-transfer process, which is the second intermediate step in the pumping process, introduces a pole at a frequency that depends on the overall lifetime of the upper laser level and on the Ho concentration in the active material. Typically this pole is located a few decades below the ROF, and hence strongly reduces the contribution of pump fluctuations, as well as of pump feedback, on the laser intensity noise at higher frequencies. Therefore, a feedback circuit acting on the pump power needs a particularly high gain at around the ROF, in order to achieve an effective suppression of the noise peak. Taking the appropriate numerical values [7] for the laser parameters, it turns out that in our case the ROF can vary between 80 and $110 \mathrm{kHz}$ while the low-frequency pole due to the energy-transfer process is located at $\sim 1 \mathrm{kHz}[5,6]$. The corresponding magnitude and phase of the calculated PTF are shown in Fig. $1 a$ and $b$, respectively. 